\title{
Dissecting the specificity of a TCR-mimic antibody targeting TP53 R175H mutation-derived neoantigen
}

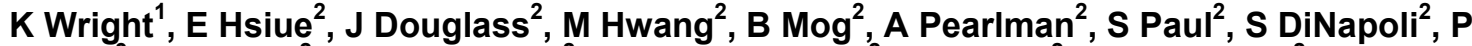 \\ Azurmendi $^{3}$, D PardolI ${ }^{2}$, C Bettegowda ${ }^{2}$, N Papadopoulos ${ }^{2}, K_{\text {Kinzler }}{ }^{2}$, B Vogelstein ${ }^{2}, S$ Gabelli $^{1,3}, S$ \\ Zhou $^{2}$ \\ ${ }^{1}$ Department of Biophysics and Biophysical Chemistry, Baltimore, MD, ${ }^{2}$ Sidney Kimmel \\ Comprehensive Cancer Center, Baltimore, MD, ${ }^{3}$ Department of Medicine, Baltimore, MD \\ kwrigh53@jhmi.edu
}

The emergence of immunotherapy as an important tool in the fight against cancer takes advantage of the exquisite specificity of antibodies. Targets, however, have been limited to those on the cell surface, despite most oncogenic driver mutations occurring in genes which encode intracellular proteins; known as "undruggable" targets. To overcome this limitation, antibodies can be selectively engineered to target mutation-derived neoantigens, peptides derived from mutant proteins that are presented on the cell surface by the Major Histocompatibility Complex Class I (MHC-I), and not their wild-type peptide-MHC counterparts. One such "undruggable" target is tumor-suppressor protein TP53, one of the most commonly mutated driver genes in all cancers. Here, we describe the identification and structural basis of a T cell receptor (TCR)-mimic antibody against the HLA-A*02:01-restricted TP53 R175H epitope, highlighting its specificity. We have developed a TCR-mimic antibody through phage display, selecting the antibody that showed specificity and selectivity toward the mutant peptide TP53 R175H over the wild-type peptide. The TP53 R175H specific antibody was expressed in mammalian cells to generate an optimal protein fold and the antibody Fab fragments were generated using enzymatic cleavage techniques. We performed both structural and biophysical characterization. Structural studies of the Fab/TP53 R175H peptide-MHC complex were carried out using X-ray crystallography to fully understand the interaction between the antibody and TP53 peptide. Furthermore, biophysical characterization included carrying out binding kinetics experiments using Surface Plasmon Resonance (SPR). Determination of the structure revealed the TCR-mimic antibody forms a cage-like configuration around the C-terminal of the TP53 R175H neoantigen, trapping residue H175 into an exposed position by providing a stable interaction. Specifically, all CDRs of the variable heavy chain interact with the displayed TP53 R175H neoantigen. In contrast, only the third CDR of the variable light chain contributes to peptide interaction. Interestingly, the TCR-mimic antibody interaction employs a non-canonical parallel binding mode, different from the typical diagonal orientation utilized by most TCRs and other TCR-mimics. It is possible this new mode of binding contributes to the observed antibody specificity and measured affinity $(\mathrm{KD}=86 \mathrm{nM})$. Exploitation of our detailed structural understanding of the mechanisms of specificity is essential for the development of more potent and selective antibodies. The exquisite selectivity and specificity achievable with antibodies provides the added benefit of distinguishing between wild-type and mutant proteins-the foundation for developing effective treatments with minimal adverse effects to patients. Our study provides a new immunotherapeutic approach to treat cancers with "undruggable" driver alterations.

Acta Cryst. (2020). A76, a57 